\title{
Uso problemático de la tecnología, motivación y rendimiento académico en escolares
}

\author{
Problematic use of technology, motivation and academic \\ performance in schoolchildren
}

TANIA LIZBETH GUTIÉRREZ LUGO

Instituto Tecnológico de Sonora, Sonora, México(tania.gutierrez@potros.itson.edu.mx)(https:// orcid.org/0000-0002-5695-4300)

DRA. MIRSHA ALICIA SOTELO CASTILLO

Instituto Tecnológico de Sonora, Sonora, México(mirsha.sotelo@itson.edu.mx)(https://orcid. org/0000-0001-9838-189X )

DRA. DORA YOLANDA RAMOS ESTRADA

"Instituto Tecnológico de Sonora, Sonora, México(dramos@itson.edu.mx)(https://orcid org/0000-0001-7933-3753)"

\section{RESUMEN}

La implementación y uso de la tecnología ha tenido beneficios importantes en el quehacer diario, sin embargo, es importante considerar que su uso también puede tener consecuencias negativas, además de representar posibles riesgos y amenazas, sobre todo en los menores. Su uso problemático puede generar una disminución en el tiempo dedicado a los estudios y con ello verse afectado los niveles de motivación hacia el aprendizaje y el rendimiento académico. El objetivo general del estudio fue determinar la relación entre el uso problemático de las tecnologías, la motivación hacia el aprendizaje y el rendimiento académico en niños y niñas escolares entre 9 y 12 años. Fue de tipo cuantitativo con diseño no experimental transversal correlacional y comparativo. Participaron 171 alumnos entre los 9 y 12 años de edad; para la recolección de los datos se utilizaron dos escalas tipo Likert relacionadas con el uso problemático de la tecnología (internet, videojuegos, celular, tablet y computadora), y la motivación hacia el aprendizaje, para la medición del rendimiento académico se consideró el promedio de calificaciones. Los resultados indicaron relaciones estadísticamente significativas entre el uso de la tecnología con el rendimiento académico y este a su vez con la motivación, asimismo, se encontraron diferencias entre los grupos de promedio bajo y alto. Es una realidad que desde edades tempranas se accede al mundo tecnológico y los convierte en un grupo vulnerable para desencadenar usos problemáticos, lo cual se ve reflejado en su desempeño. 


\begin{abstract}
The implementation and use of technology has had important benefits in daily life, however, it is important to consider that its use can also have negative consequences, in addition to representing possible risks and threats, especially in children. Its problematic use can generate a decrease in the time dedicated to studies and thus affect the levels of motivation towards learning and academic performance. The objective of the study was to determine the relationship between the problematic use of technologies, learning motivation and academic performance in school children between 9 and 12 years of age. The study was quantitative with a non-experimental cross-sectional correlational and comparative design. A total of 171 students between 9 and 12 years of age participated; two Likert-type scales related to the problematic use of technology (internet, video games, cell phone, tablet and computer) and the motivation of their learning process were used for data collection; for the measurement of academic performance, the grade point average was considered. The results indicated statistically significant relationships between the use of technology with academic performance and this in turn with motivation, likewise, differences were found between the low and high average groups. It is a reality that from an early age students have access to the technological world and this makes them a vulnerable group to trigger problematic uses, which is reflected in their performance.
\end{abstract}

\title{
PALABRAS CLAVE / KEYWORDS
}

Information technology, motivation, academic achievement, students, elementary level, learning. / Tecnología de la información, motivación, rendimiento escolar, estudiantes, nivel primaria, aprendizaje.

\section{INTRODUCCIÓN}

Las Tecnologías de la Información y la Comunicación (TIC) han transformado de manera acelerada la vida cotidiana y social de los seres humanos; Sánchez (2018) hace mención que son necesarias para gestionar y transformar la información que se maneja día a día con apoyo de ordenadores y programas específicos para ello; Adell (2006) sostiene que es el "conjunto de procesos y productos derivados de las nuevas herramientas (hardware y software), soportes de la información y canales de comunicación relacionados con el almacenamiento, procesamiento y transmisión digitalizados de la información" (p.7).

El usar las tecnologías no es bueno ni malo, todo depende del uso que se haga de ellas, de hecho, su implementación ha generado rápidos e importantes cambios en la sociedad, beneficiando la calidad de vida de las personas; sin embargo, no todo es bueno, ya que su uso también puede generar consecuencias negativas, además de representar posibles riesgos y amenazas para las personas (Duarte, 2008). Su adecuada utilización genera importantes 
beneficios, pero también parece conllevar problemas. El aspecto más controvertido y que causa mayor alarma social, es el potencial adictivo y uso problemático de estas tecnologías, especialmente entre los menores, quienes son los que mayor uso hacen de ellas (Labrador \& Villadangos, 2010); especialmente se observa un uso excesivo en los dispositivos móviles y sus aplicaciones, tales como la mensajería instantánea, los videojuegos, el internet y las redes sociales. Los y las menores sienten verdadera atracción por todo lo relacionado con las tecnologías, ya que la evidencia demuestra que su uso puede satisfacer algunas necesidades, tales como de entretenimiento, diversión, comunicación e información (Asociación de cooperación para el desarrollo y la seguridad digital, 2019).

Diversas investigaciones han trabajado sobre este tema, al respecto en la investigación realizada por Díaz-Vicario, et al. (2020), se estudió el uso problemático de las tecnologías en el contexto académico, social y familiar. De acuerdo a los resultados encontrados, se confirma que las TIC pueden generar una disminución del tiempo dedicado al estudio y por consiguiente afectar el rendimiento académico del alumno. Además, se evidencia el surgimiento de conflictos familiares a causa del uso excesivo y/o por hacer uso de las TIC mientras se realizan actividades en familia.

En el estudio realizado por Muñoz-Miralles, et al. (2013) relacionada con el fracaso escolar y el uso excesivo del internet, se observó que los y las estudiantes que pasan mayor tiempo frente al ordenador, sin la supervisión de sus familias son los que obtienen bajos resultados escolares. Además, se hace referencia al uso del ordenador con finalidades lúdicas, lo que posiblemente afecte el rendimiento académico del alumno y por consiguiente lo pone en un mayor riesgo de fracaso escolar.

Pauta (2015) en su investigación sobre los niños y la influencia de la televisión y rendimiento escolar, en una muestra conformada por 219 alumnos y alumnas de educación primaria, concluyó que las horas que los niños y niñas dedican a ver televisión no afecta su rendimiento académico, ya que los padres de familia los controlan a la hora de hacer sus tareas y ver televisión.

Sin embargo, Fuentes y Pérez (2015) en su investigación sobre los videojuegos y sus efectos escolares, conformada por 480 estudiantes, concluye que en los y las escolares que practican videojuegos su rendimiento académico ha disminuido, ya que la mayoría de los encuestados han perdido asignaturas después de ser usuarios de videojuegos. Además, reemplazan actividades como deporte, paseos y tareas escolares, lo que conlleva a disminuir el rendimiento académico y la generación de conductas tales como adicción, agresividad y aislamiento.

El estudio realizado por Llorca, et al., (2010), sobre la frecuencia en el uso de videojuegos y la relación con el rendimiento académico en una población de 266 estudiantes con edades entre los 11 y 16 años, muestra que casi un tercio de la muestra juegan solo los fines de semana y un menor porcentaje juegan todos los días concluyendo que efectivamente la moderación o el uso controlado de los videojuegos es una variable con efecto sobre el rendimiento académico de los alumnos.

Por otra parte, otra variable asociada al uso de la tecnología ha sido la motivación hacia el aprendizaje. Buñuelos (1993) define la motivación como un proceso general por el cual se inicia y dirige una conducta hacia el logro de una meta. Este proceso involucra variables tanto afectivas como cognitivas, en cuanto a habilidades de pensamiento y 
conductas instrumentales para alcanzar las metas propuestas. Valenzuela (2007) sostiene que la motivación escolar tiene que ver más concretamente con la activación de recursos cognitivos, para aprender aquello que la escuela propone como aprendizaje. Bravo, et al. (2019) hacen referencia que la motivación "es un concepto basado en la emoción que mueve a las personas a hacer cosas, el motivo que lo lleva a realizar una acción dependiendo que gustos e intereses pueda tener en ese momento" (p.20).

Al analizar las diferentes definiciones del concepto de motivación se encuentran diferencias entre ellas, mismas que se deben a las diferentes posturas teóricas y los factores que intervienen en ella (Antolín, 2013), no obstante, independiente del referente teórico, la motivación es importante para el estudiante por ser un elemento necesario para alcanzar el éxito, ya que es indispensable por ser la encargada de mover al alumnado y permitirle que alcance sus objetivos. De igual manera, siempre se encontrará sometida a diversos estímulos, ya sean internos o externos, por lo que el estudiante deberá saber cómo tomar a cada uno de estos y manejarlos según el momento en el que se encuentre, con tal de evitar que lleguen a afectarlo y se conviertan en un obstáculo para cumplir con sus metas (Llanga, et al., 2019), y uno de estos elementos puede ser el uso excesivo o mal uso de las tecnologías.

Al respecto se han encontrado diferentes hallazgos; Solís y Valdivia (2018) concluyen que existe una relación significativa positiva, aunque con intensidad baja entre motivación y uso de tecnología. Esto puede ser debido a que existen muchos factores que influyen en la motivación para el aprendizaje del estudiante, y las TIC serían solo una parte. Asimismo, Huertas y Pantoja (2016) realizaron una investigación sobre los efectos de un programa educativo basado en el uso de las tecnologías en el rendimiento académico y la motivación de los y las estudiantes; los resultados de la investigación indicaron que los niños y niñas que emplearon las tecnologías obtuvieron mejores calificaciones y mostraron mayores niveles de motivación. Sin embargo, es posible que el uso de la tecnología sin fines educativos repercute en la motivación y por ende en el rendimiento académico del alumno cuando no hay control en el uso.

\section{PLANTEAMIENTO DEL PROBLEMA}

Ha sido tanto el impacto que la tecnología ha tenido en la sociedad, que actualmente el entorno de los niños llega a determinar el uso y abuso que los infantes y adolescentes hacen de ella (Sánchez-Teruel, et al., 2016). La revisión de literatura evidencia que el rendimiento académico es atribuido a factores relacionados con el estudiante, por ejemplo, el género; factores psicosociales como motivación y la ansiedad, factores relacionados con la escuela, como infraestructura escolar, los docentes, relación entre pares; la familia, por ejemplo el nivel socioeconómico, y entre muchos otros el uso que hacen de la tecnología (Heredia \& Camacho, 2014).

Con respecto a esto último, Roca (2015) menciona que dentro de las desventajas del uso excesivo de las tecnologías, fomenta el ser una persona pasiva, al no interactuar con otros, teniendo como resultado niños y niñas socialmente aislados, y que de cierta forma tienden a presentar problemas para desarrollar relaciones con otras personas, existe menos actividad física y por consecuencia mayores índices de enfermedades; el uso descontrolado de la 
tecnología puede causar hiperactividad, irritabilidad y depresión infantil. De igual manera, Sánchez, et al. (2015), mencionan que el dedicar mucho tiempo al uso de juegos o redes sociales puede afectar al estudio y generar poco interés e incluso abandonar otras actividades sociales, culturales, deportivas. Es por ello que a partir de lo anterior se plantea la siguiente pregunta de investigación: ¿cómo se asocia el uso problemático de las tecnologías con la motivación hacia el aprendizaje y el rendimiento académico en niños y niñas de educación primaria con edades entre los 9 y 12 años de edad?

\section{OBJETIVOS}

El objetivo general del estudio fue determinar la relación entre el uso problemático de las tecnologías, la motivación hacia el aprendizaje y el rendimiento académico en niños y niñas escolares entre 9 y 12 años.

\section{OBJETIVOS ESPECÍFICOS:}

- Describir la frecuencia de uso que hacen los niños y niñas escolares entre 9 y 12 años de las tecnologías.

- Describir el nivel de motivación hacia el aprendizaje que presentan los niños y niñas escolares entre 9 y 12 años.

- Describir el rendimiento académico que tienen los niños y niñas escolares entre 9 y 12 años.

- Comparar las variables de estudio por sexo y promedio (alto, medio y bajo).

\section{MÉTODO}

El diseño de la investigación es cuantitativa no experimental transversal con alcance correlacional y comparativo, ya que los datos se recolectaron en un solo momento, se establecieron relaciones entre las variables de estudio y se realizaron comparaciones por sexo y estudiantes con alto, medio y bajo promedio.

Muestra: Participaron un total de 171 alumnos, de los cuales 85 son mujeres y 86 son hombres. La selección de la muestra se llevó a cabo mediante el método no probabilístico por conveniencia. Los participantes estaban inscritos en los grados: cuarto, quinto y sexto de educación primaria, con edades entre los 9 y 12 años.

Instrumentos: Para la recolección de los datos se utilizó el cuestionario de Uso Problemático de Nuevas Tecnologías (UPNT), desarrollado por los autores Labrador, et al. (2013), el cual está conformado por 49 ítems con escala tipo Likert con 4 opciones de respuesta (Nunca-Siempre). Los reactivos están distribuidos en 3 dimensiones: 1) uso del internet, 2) uso de videojuegos $y, 3)$ uso del celular, tablet, computadora. Para la muestra de estudio se obtuvo un índice de fiabilidad de alfa de Cronbach de .905, lo que indica un alto nivel de confianza (Hair, et 
al., 1999). Adicionalmente se hicieron preguntas sobre la frecuencia de uso y problemas de dichas tecnologías, y la frecuencia en que su uso le genera problemas.

Por otra parte, para medir la motivación hacia el aprendizaje se utilizó el Cuestionario de evaluación motivacional del proceso de aprendizaje (EMPA), desarrollado por QuevedoBlasco y Téllez-Trani (2016). Consta de 33 ítems con dos subescalas que proporcionan información relativa a la motivación intrínseca y extrínseca. Está diseñado bajo una escala tipo Likert, con cinco opciones de respuesta que van desde casi nada, un poco, algunas veces, casi siempre y siempre. Para la muestra de estudio se obtuvo un índice de fiabilidad de alfa de Cronbach de .942, lo que indica un alto nivel de confianza (Hair, et al., 1999).

Para la medición del rendimiento académico se preguntó directamente el promedio de calificaciones obtenido a la fecha del levantamiento de los datos.

Procedimiento: Primeramente, se contactó con el director de la escuela primaria seleccionada para la recolección de los datos, se le comunicó el objetivo de la investigación y se le solicitó la autorización para la aplicación de los instrumentos a los alumnos requeridos según los criterios de inclusión (escolares de 9 a 12 años). Una vez obtenida la autorización se acudió a los grupos para la aplicación de los instrumentos, mismos que contaban con un consentimiento informado, el cual postulaba el objetivo de la investigación y mencionaba que los participantes estaban en total disposición de cooperar contestando el instrumento con una duración de 40 minutos. Además, se les informó que la información obtenida del estudio era totalmente confidencial, y que esta quedaría bajo reserva del investigador y que tenían el derecho de conocer los resultados si así lo solicitaban.

Finalmente una vez terminada la aplicación, se capturaron los datos en el programa estadístico SPSS versión 23. Para el análisis de los datos se utilizó estadística paramétrica, ya que los datos mostraron una distribución normal, según los índices de asimetría y curtosis. Para la correlación se utilizó la prueba r de Pearson para establecer una relación entre el uso problemático de la tecnología, motivación escolar y rendimiento académico, y por último para las comparaciones se utilizó la prueba t de Student para muestras independientes, para comparar hombres y mujeres y ANOVA para comparar los grupos de promedios: alto, medio y bajo.

\section{RESULTADOS}

En el uso problemático del Internet, los niños indicaron que a veces su uso si genera problemas en ellos, se obtuvo una media 2.25 (DE=.665), considerando una escala de 1 al 4 . El indicador que obtuvo una media más alta fue, que ellos creen que dedican más tiempo del necesario en internet ( $M=2.66)$. Sin embargo, también mencionan que navegar por internet, no es problema, sino que se sienten relajados $(\mathrm{M}=2.88)$ (ver tabla 1). 


\begin{tabular}{|c|c|c|c|c|}
\hline Indicador & Min & Max & $\mathbf{M}$ & DE \\
\hline ¿Dedicas más tiempo del que crees necesario a internet? & 1 & 4 & 2.66 & 1.118 \\
\hline ¿Dedicas más tiempo del que crees necesario a internet? & 1 & 4 & 2.66 & 1.118 \\
\hline $\begin{array}{l}\text { ¿Te encuentras mal cuando por algún motivo no puedes utilizar internet } \\
\text { queriendo hacerlo? }\end{array}$ & 1 & 4 & 2.04 & 1.158 \\
\hline ¿Estás pensando desde horas antes de conectarte a internet en ello? & 1 & 4 & 2.55 & 1.302 \\
\hline ¿Mientes a tu familia o amigos sobre las horas que dedicas a utilizar internet? & 1 & 4 & 1.54 & .941 \\
\hline ¿Alguna vez has intentado desconectarte de internet y no lo has conseguido? & 1 & 4 & 2.16 & 1.180 \\
\hline ¿Te relaja navegar por internet? & 1 & 4 & 2.88 & 1.113 \\
\hline $\begin{array}{l}\text { ¿Te sientes nervioso si pasa mucho tiempo desde la última vez que estuviste } \\
\text { conectado a internet? }\end{array}$ & 1 & 4 & 1.96 & 1.229 \\
\hline
\end{tabular}

En la escala del uso problemático de los videojuegos los niños presentaron una media de 1.77 ( $D E=1.084$ ) que corresponde a un nivel en donde los niños de acuerdo a su criterio subjetivo nunca presentan problemas con el uso de los videojuegos. El indicador que obtuvo una media más alta fue que los niños desde horas antes están pensando en jugar videojuegos ( $M=1.97)$. Sin embargo, los niños lo atribuyen a que jugar videojuegos los relaja $(M=2.34)$ (ver tabla 2).

\begin{tabular}{|c|c|c|c|c|}
\hline Indicador & Min & Max & M & DE \\
\hline ¿Dedicas más tiempo del que crees necesario a los videojuegos? & 1 & 4 & 1.75 & 1.028 \\
\hline $\begin{array}{l}\text { ¿Te encuentras mal cuando por algún motivo no puedes jugar a los video- } \\
\text { juegos? }\end{array}$ & 1 & 4 & 1.81 & 1.092 \\
\hline ¿Estás pensando desde horas antes de jugar con los videojuegos? & 1 & 4 & 1.97 & 1.190 \\
\hline $\begin{array}{l}\text { ¿Mientes a tu familia o amigos sobre las horas que dedicas a jugar a los vi- } \\
\text { deojuegos? }\end{array}$ & 1 & 4 & 1.39 & .829 \\
\hline ¿Alguna vez has intentado dejar de jugar y no lo has conseguido? & 1 & 4 & 1.71 & 1.009 \\
\hline ¿Te relaja jugar a los videojuegos? & 1 & 4 & 2.34 & 1.213 \\
\hline ¿Te sientes nervioso si pasa mucho tiempo desde la última vez que jugaste? & 1 & 4 & 1.61 & 1.048 \\
\hline
\end{tabular}

En la escala del uso problemático del uso del celular, tablet, iPad, computadora etc. Los niños presentaron una media de 1.87 ( $\mathrm{DE}=.943$ ), que corresponde a un nivel en donde los niños de acuerdo a su criterio subjetivo nunca han presentado problemas con el uso de los aparatos electrónicos con acceso a internet. El indicador que tuvo una media más alta fue que los niños están pensando desde horas antes en hacer uso del celular, Tablet, computadora, etc. (M=2.27). Sin embargo, los niños lo atribuyen a que hacer uso de aparatos electrónicos con acceso a internet los relaja ( $\mathrm{M}=2.61)$ (ver tabla 3). 


\begin{tabular}{|c|c|c|c|c|}
\hline Indicador & Min & Max & M & DE \\
\hline $\begin{array}{l}\text { ¿Te encuentras mal cuando por algún motivo no puedes usar el Celular, Ta- } \\
\text { blet, IPad, Computadora, Laptop o IPod? }\end{array}$ & 1 & 4 & 1.94 & 1.069 \\
\hline $\begin{array}{l}\text { ¿Estás pensando desde horas antes de usar el Celular, Tablet, IPad, Compu- } \\
\text { tadora, Laptop o IPod? }\end{array}$ & 1 & 4 & 2.27 & 1.193 \\
\hline $\begin{array}{l}\text { ¿Mientes a tu familia o amigos sobre las horas que dedicas al Celular, Tablet, } \\
\text { IPad, Computadora, Laptop o IPod? }\end{array}$ & 1 & 4 & 1.40 & .755 \\
\hline $\begin{array}{l}\text { ¿Alguna vez has intentado dejar de usar el Celular, Tablet, IPad, Computado- } \\
\text { ra, Laptop o IPod y no lo has conseguido? }\end{array}$ & 1 & 4 & 2.05 & 1.134 \\
\hline ¿Te relaja usar el Celular, Tablet, IPad, Computadora, Laptop o IPod? & 1 & 4 & 2.61 & 1.185 \\
\hline $\begin{array}{l}\text { ¿Te sientes nervioso si pasa mucho tiempo desde la última vez que usaste el } \\
\text { Celular, Tablet, IPad, Computadora, Laptop o IPod? }\end{array}$ & 1 & 4 & 1.70 & 1.040 \\
\hline
\end{tabular}

De acuerdo a los resultados obtenidos en la variable de motivación, se obtuvo una media total de 3.89 ( $D E=.761$ ), en una escala del 1 al 5 indica que los estudiantes de manera general presentan una motivación moderada, es decir tienen buenas intenciones al estudio. Las medias fueron clasificadas, considerando la amplitud del rango, en tres niveles de motivación: alta, media y baja, resultando que un $30.4 \%$ de los alumnos presentaron una motivación entre media y baja. Al analizar las dimensiones de la escala de motivación, se encontró que en la motivación extrínseca los estudiantes obtuvieron una media general de 2.52 ( $D E=.474$ ) lo que indica que los niños presentan una motivación extrínseca moderada, es decir que le resultan moderadamente atractivos los refuerzos positivos como negativos provenientes del exterior. Se encontró que el $43.3 \%$ de los alumnos presentan una motivación extrínseca entre media y baja. En lo que respecta a la motivación intrínseca, los estudiantes presentaron una media de $4(\mathrm{DE}=.770)$, lo que indica que presentan un nivel alto de motivación, es decir realizan acciones por la mera satisfacción de hacerlas sin necesidad de algún incentivo externo. Se encontró que el $26.9 \%$ de los alumnos presentan una motivación intrínseca entre media y baja (ver tabla 4).

\begin{tabular}{|c|c|c|c|c|c|c|}
\hline \multicolumn{7}{|c|}{ Tabla 4: Rangos de motivación total } \\
\hline \multirow[t]{2}{*}{ Nivel } & \multicolumn{2}{|c|}{ Motivación total } & \multicolumn{2}{|c|}{ Motivación extrínseca } & \multicolumn{2}{|c|}{ Motivación intrínseca } \\
\hline & fr & $\%$ & fr & $\%$ & fr & $\%$ \\
\hline Baja motivación & 10 & 5.8 & 15 & 8.8 & 6 & 3.5 \\
\hline Moderada motivación & 42 & 24.6 & 59 & 34.5 & 40 & 23.4 \\
\hline Alta motivación & 119 & 69.6 & 97 & 56.7 & 125 & 73.1 \\
\hline
\end{tabular}

En cuanto a la variable de rendimiento académico se dividió en los siguientes rangos: bajo (6 a 7.99), promedio (8 a 8.99) y alto (9 a 10), los porcentajes de distribución fueron 12.9, 33.3, 53.8 respectivamente. Los estudiantes obtuvieron una media de 8.75 , lo que indica que los alumnos se encuentran en un nivel promedio, sin embargo son más los escolares que se ubicaron en el rango alto. 


\section{CORRELACIONES ENTRE VARIABLES}

De acuerdo a los resultados encontrados en los índices de correlación entre las variables de estudio, se puede observar que las variables relacionadas con el uso problemático de las tecnologías, que son el uso de internet, uso de videojuegos, uso del celular, tablet, computadora, etc., obtuvieron una correlación estadísticamente significativa y negativa con el rendimiento académico, lo cual pudiera significar que a mayor uso de las tecnologías el promedio del alumno tiende a bajar. Asimismo, la motivación también correlacionó significativamente con el rendimiento pero de forma positiva, lo cual es probable que a mayor motivación hacia los estudios, mayor será el promedio del alumno o entre mayor sea el promedio académico del alumno mayor será su motivación hacia los estudios (ver tabla 5).

Por otra parte, la motivación del alumno hacia los estudios no presentó relación significativa, es decir cuando se trata de usar los diferentes dispositivos, lo cual puede significar que los niños pueden estar motivados y hacer uso del internet, los videojuegos, del celular, tablet y computadora etc., o pueden estar no motivados y aun así seguir haciendo uso de los medios antes mencionados.

\begin{tabular}{|l|l|l|l|l|l|l|}
\hline \multicolumn{7}{|c|}{ Tabla 5: Correlación de las variables de estudio } \\
\hline \multicolumn{1}{|c|}{ Indicador } & Promedio & $\begin{array}{c}\text { Uso de } \\
\text { internet }\end{array}$ & $\begin{array}{c}\text { Uso de vi- } \\
\text { deojuegos }\end{array}$ & $\begin{array}{c}\text { Uso de celular, tablet, } \\
\text { computadora }\end{array}$ & Motivación \\
\hline Promedio & 1 & & & & \\
\hline Uso de internet & $-.246^{* *}$ & 1 & & & \\
\hline Uso de videojuegos & $-.305^{* *}$ & $.574^{* *}$ & 1 & & \\
\hline $\begin{array}{l}\text { Uso de celular, tablet, com- } \\
\text { putadora }\end{array}$ & $-.329^{* *}$ & $.675^{* *}$ & $.610^{* *}$ & 1 & \\
\hline Motivación & $.438^{* *}$ & -.150 & -.110 & -.129 & 1 \\
\hline
\end{tabular}

\section{RESULTADOS DE COMPARACIONES ENTRE SEXO}

Para realizar la comparación se utilizó la prueba t de Student para muestras independientes, los resultados mostraron una diferencia estadísticamente significativa en la variable uso problemático de los videojuegos en relación con el sexo de los estudiantes, donde el sexo masculino obtuvo una media más alta $(M=2.08)$ que las mujeres $(M=1.45)$, esto quiere decir que no solo los niños hacen mayor uso de los videojuegos sino también suele tener mayor problemas a causa de su uso, con un tamaño del efecto mediano ( $d=$ .611). Además, se encontró una diferencia estadísticamente significativa en la variable de motivación extrínseca en relación con el sexo de los estudiantes, donde el sexo masculino obtuvo una media más alta de $(M=2.59)$ que el sexo femenino de $(M=2.44)$, con un tamaño del efecto pequeño $(d=.214)$, esto quiere decir que los niños son los que presentan mayor 
motivación extrínseca que las niñas (ver tabla 6).

\begin{tabular}{|c|c|c|c|c|c|c|c|c|}
\hline \multicolumn{9}{|c|}{ Tabla 6: Comparación por sexo de las variables estudiadas } \\
\hline & \multicolumn{2}{|c|}{ Masculino } & \multicolumn{2}{|c|}{ Femenino } & \multirow[t]{2}{*}{ gl } & \multirow[t]{2}{*}{$\mathbf{t}$} & \multirow[t]{2}{*}{$\mathbf{p}$} & \multirow{2}{*}{$\begin{array}{l}\text { d de } \\
\text { Cohen }\end{array}$} \\
\hline & M & DE & M & DE & & & & \\
\hline Uso de internet & 2.28 & .71 & 2.22 & .61 & 165.5 & .610 & .54 & .090 \\
\hline Uso de videojuegos & 2.08 & 1.18 & 1.45 & .88 & 157.2 & 3.913 & .00 & .611 \\
\hline $\begin{array}{l}\text { Uso del celular, Tablet, com- } \\
\text { putadora }\end{array}$ & 1.87 & 1.01 & 1.87 & .87 & 166.1 & .010 & .99 & .000 \\
\hline otivación total & 3.84 & .80 & 3.93 & .72 & 167.6 & -.829 & .40 & .118 \\
\hline Motivación intrínseca & 3.94 & .79 & 4.05 & .74 & 168.5 & -.928 & .35 & .156 \\
\hline Motivación extrínseca & 2.59 & .47 & 2.44 & .46 & 169 & 2.163 & .03 & .214 \\
\hline
\end{tabular}

Al comparar las variables por grupo de promedio se utilizó la prueba ANOVA de un factor, y se encontró una diferencia estadísticamente significativa entre los grupos en el uso de internet, videojuegos y celular, tablet y computadora, siendo los alumnos con promedio bajo los que hacen la diferencia. También, se encontró una diferencia estadísticamente significativa entre grupos en la variable de motivación y en la dimensión de motivación intrínseca y extrínseca, observando que son los alumnos con bajo promedio los que presentan menor motivación general, extrínseca e intrínseca, las diferencias significativas presentaron un moderado tamaño del efecto (ver tabla 7).

\begin{tabular}{|c|c|c|c|c|c|c|c|c|c|c|}
\hline & \multicolumn{2}{|c|}{ Promedio bajo } & \multicolumn{2}{|c|}{ Promedio regular } & \multicolumn{2}{|c|}{ Promedio alto } & \multirow[t]{2}{*}{ gl } & \multirow[t]{2}{*}{$\mathbf{F}$} & \multirow[t]{2}{*}{$\mathbf{p}$} & \multirow[t]{2}{*}{$\mathbf{f}$} \\
\hline & M & DE & $\mathbf{M}$ & DE & $\mathbf{M}$ & DE & & & & \\
\hline Uso de internet & 2.49 & .60 & 2.40 & .62 & 2.10 & .67 & 170 & 5.30 & .006 & .260 \\
\hline $\begin{array}{l}\text { Uso del celular, Ta- } \\
\text { blet, computadora }\end{array}$ & 2.40 & 1.07 & 2.04 & 1.00 & 1.64 & .79 & 170 & 7.68 & .001 & .285 \\
\hline Motivación total & 3.39 & .97 & 3.65 & .86 & 4.15 & .48 & 170 & 15.37 & .000 & .384 \\
\hline $\begin{array}{l}\text { Motivación intrín- } \\
\text { seca }\end{array}$ & 3.48 & .98 & 3.74 & .85 & 4.28 & .49 & 170 & 16.99 & .000 & .408 \\
\hline $\begin{array}{l}\text { Motivación extrín- } \\
\text { seca }\end{array}$ & 2.63 & .39 & 2.63 & .41 & 2.42 & .50 & 170 & 4.58 & .012 & .241 \\
\hline
\end{tabular}




\section{DISCUSIÓN}

De acuerdo a los resultados de la investigación en cuanto a la variable uso problemático de las tecnologías al usar internet, videojuegos, celular, tablet y computadora, se concluye que la muestra de estudio no percibe problemas en el uso de estas tecnologías, a excepción del uso del internet, ya que los escolares consideraron que a veces suele tener problemas a causa de su uso. Estos resultados difieren por los encontrados por García-Oliva, et al. (2017) quienes concluyen que su población de estudio presenta niveles elevados de uso problemático de internet, uso del celular y uso de videojuegos.

Es importante mencionar que de acuerdo al criterio de los niños no presentan problemas con el uso de los videojuegos, pero desde horas antes están pensando en ello, lo mismo pasa con el uso del celular, tablet, y computadora justificando que su uso los relaja. Lo anterior coincide con Labrador y Villadangos (2010) donde la conducta más significativa aparece en todas las nuevas tecnologías y con las mayores puntuaciones en la relajación de su uso, lo que se concluye que es posible que esta inducción de relajación sea similar al efecto que el consumo de sustancias produce en personas adictas 0 , simplemente, que se usan las nuevas tecnologías como conductas de ocio.

Los niños a diario hacen uso de las tecnologías antes mencionadas entre 1 y 4 horas al día, dato que está ligeramente superior al que presentan otros estudios que sitúan la media en 3 horas al día (Díaz-Vicario, et al., 2020). En relación con los lugares de conexión, el mayor porcentaje recae en el hogar.

En cuanto a la variable de motivación los alumnos de manera general presentan una motivación moderada, es decir tiene motivación hacia su proceso de aprendizaje, lo que coincide con los resultados presentados por Antolín (2013) donde su población presentó una motivación general entre media y alta a los estudios. Finalmente en lo que respecta a la motivación intrínseca los alumnos presentan un nivel alto, es decir realizan acciones por la sola satisfacción de hacerlas sin necesidad de algún incentivo externo, lo cual coincide con Usán y Salavera (2018) en donde su población de estudio mostró mayor motivación hacia el conocimiento y hacia el logro.

Por otra parte, se comprobaron las hipótesis de relación entre el uso de las diferentes tecnologías cuestionadas con el rendimiento académico, pudiendo concluir que es altamente probable que a mayor uso de las tecnologías el promedio del alumno tienda a bajar, esto coincide con lo reportado por Badia, et al. (2015) quienes han concluido que el mal uso de las tecnologías puede repercutir en un menor rendimiento académico del alumno.

Asimismo, la motivación también correlacionó significativamente con el rendimiento pero de forma positiva, lo cual es probable que a mayor motivación hacia los estudios mayor será el promedio del alumno o entre mayor sea el promedio académico del alumno mayor será su motivación hacia su propio aprendizaje. La intensidad de la relación fue moderada, lo cual coincide con lo reportado por Antolín (2013).

La motivación del alumno hacia los estudios no presentó relación significativa, es decir cuando se trata de usar los diferentes dispositivos, lo cual puede significar que los niños pueden estar motivados y hacer uso del internet, los videojuegos, del celular, tablet, computadora, etc. o pueden estar no motivadas y aun así seguir haciendo uso de los medios 
antes mencionados. En contraste, en la investigación realizada por Huertas y Pantoja (2016) se encontró que los alumnos que emplean las TIC obtienen mejores calificaciones y están más motivados.

En cuanto a las hipótesis de comparación planteadas se comprobaron, ya que se encontraron diferencias significativas en la variable uso problemático de los videojuegos en relación con el sexo de los estudiantes, donde el sexo masculino hace mayor uso de los videojuegos que el sexo femenino y además suele presentar mayores problemas a causa de su uso. Coincidiendo con los resultados de Castellana, et al. (2007) en donde los hombres están más interesados y hacen mayor uso de los videojuegos que las mujeres, mientras que en la investigación realizada por García-Oliva, et al. (2017) la combinación de uso de internet y móvil es más problemática en las chicas y el uso de internet y videojuegos es más problemático en los chicos.

También, se comprobó una diferencia significativa en la variable de motivación extrínseca en relación con el sexo de los estudiantes. Los niños son los que presentan mayor motivación extrínseca que las niñas.

Dentro de los datos más significativos de esta investigación fue la comprobación de las hipótesis de comparación por promedio, ya que se comprobaron diferencias entre los alumnos con promedio alto, medio y bajo con respecto al uso problemático del internet y la motivación, haciendo esta diferencia los estudiantes que mantienen un promedio bajo. Sin embargo, en el estudio de Champa (2017) sobre el uso de internet y su relación con el rendimiento académico concluyeron que los estudiantes que aseguraron utilizar Internet en sus estudios mejoraron el rendimiento escolar. De la misma manera se encontró una diferencia estadísticamente significativa entre el uso de los videojuegos, uso del celular, tablet y computadora con el rendimiento académico del alumno, concluyendo que los que hacen mayor uso de ellos son los alumnos que tienen un promedio bajo. Esto difiere de lo encontrado por Badia, et al. (2015) y Sánchez (2018) quien afirma que el uso de videojuegos y aparatos electrónicos para fines académicos logran obtener un mejor resultado y mayor promedio.

Así mismo, se encontró una diferencia estadísticamente significativa entre la variable motivación y el rendimiento académico de los alumnos. Los alumnos que presentan una mayor motivación a los estudios son los que tienen un promedio alto. De la misma manera, Antolín (2013) logró encontrar diferencias significativas en los datos de motivación, siendo significativamente menor la motivación de los alumnos que mantienen un promedio bajo.

\section{CONCLUSIÓN}

A manera de cierre, es una realidad que los niños desde edades tempranas empiezan a acceder al mundo tecnológico, lo cual los coloca como una comunidad de alto riesgo en desencadenar usos problemáticos. Retomando la respuesta a la pregunta de investigación y cumpliendo con los objetivos planteados se encontró que efectivamente existe una relación entre el uso problemático de las tecnologías con el rendimiento académico del alumno. Además se pudo observar que quienes presentan mayores problemas a causa del uso de las 
tecnologías son los alumnos con un promedio bajo.

Es importante para psicólogos e investigadores prestar más atención a las problemáticas generadas en poblaciones de menor edad, ya que es un campo de estudio olvidado y desenfocado, donde desafortunadamente se están perdiendo datos muy valiosos. Se recomienda realizar actualizaciones constantes respecto a la presente investigación, siempre utilizando recursos científicos confiables, de esta manera será posible obtener resultados más certeros y concretos, lo que facilitará la obtención de los resultados esperados.

Los hallazgos encontrados señalan la importancia de prestar mayor atención a los más pequeños, como medida preventiva a los problemas que puede generar el uso de las tecnologías, por lo que es necesario insistir en la prevención y la formación de hábitos de buen uso de las tecnologías, incluso antes de los 12 años. Asimismo, es necesario profundizar en los motivos o circunstancias en las que se produce el uso problemático de las tecnologías.

Por último, se recomienda ser cautelosos en los resultados obtenidos, ya que una de las principales limitaciones de este estudio es la imposibilidad de generalizar los resultados a la población de niños de esa edad de la muestra de estudio, debido a que los estudiantes pertenecen a una sola institución de un país, y forman parte de una muestra no probabilística. Además de presentar una desventaja frente a un estudio de tipo experimental.

\section{REFERENCIAS}

Adell, J. (2006). Tendencias en educación en la sociedad de las tecnologías de la información. EDUTEC. Revista Electrónica de Tecnología Educativa, 7. https://doi.org/10.21556/ edutec.1997.7

Antolín, R. (2013). Motivación y rendimiento escolar en educación primaria [Tesis de maestría no publicada]. Universidad de Almería. http://repositorio.ual.es/bitstream/ handle/10835/3060/Trabajo.pdf?sequence=

Asociación de cooperación para el desarrollo y la seguridad digital. (2019). Uso excesivo de las Tic en menores y adolescentes. ACDeS Digital. https://acdesdigital.org/uso-excesivode-las-tic-en-menores-y-adolescentes/

Badia, M., Clariana, M., Gotzens, C., Cladellas., R. \& Dezcallar, T. (2015). Videojuegos, televisióny rendimiento académico en alumnos de primaria. Pixel-Bit. Revista de Medios y Educación, (46), pp. 25-38. https://recyt.fecyt.es/index.php/pixel/article/view/61608/37621

Bravo, M., Ospina., H., Vázquez., H., \& Holguín, W. (2019). Factores que influyen en la motivación de un grupo de estudiantes de básica secundaria en las clases de educación física en una institución educativa del municipio de Bello [Tesis de pregrado no publicado]. Universidad de San Buenaventura Colombia. http://bibliotecadigital.usbcali.edu.co/ bitstream/10819/7389/1/Motivacion_Educacion_Fisica_Vasquez_2019.pdf

Buñuelos, A. (1993). Motivación escolar. Estudio de variables afectivas. Perfiles Educativos, 60. https://www.redalyc.org/pdf/132/13206011.pdf

Castellana, M., Sánchez-Carbonell, J. Graner, C., \& Beranuy, M. (2007). El adolescente ante las tecnologías de la información y la comunicación: internet, móvil y videojuegos. Papeles de Psicólogo, 28(3), pp. 196-204. https://www.redalyc.org/pdf/778/77828306.pdf 
Champa, R. (2017). El uso de internet y su relación con el rendimiento escolar en los estudiantes del V ciclo de educación primaria de la I.E. Experimental de Aplicación de la UNE. [Tesis de pregrado]. Universidad Nacional de Educación. https://repositorio.une. edu.pe/handle/UNE/1191

Díaz-Vicario, A., Mercader, C., \& Gairín, J. (2020). Uso problemático de las TIC en adolescentes. Revista Electrónica de Investigación Educativa, 21. https://doi.org/10.24320/redie.2019.21. e07.1882

Duarte, E. (2008). Las tecnologías de información y comunicación (tic) desde una perspectiva social. Revista Electrónica Educare, XII, pp. 155-162. https://www.redalyc.org/ pdf/1941/194114584020.pdf

Fuentes, L., \& Pérez, L. (2015). Los videojuegos y sus efectos en escolares de Sincelejo, Sucre (Colombia). Opción, 31(6), pp. 318-328. https://www.redalyc.org/pdf/310/31045571020. pdf

García-Oliva, C., Piqueras., J., \& Marzo, J. (2017). Uso problemático de internet, el móvil y los videojuegos en una muestra de adolescentes alicantinos. Salud y Drogas, 17(2), pp. 189200. https://www.redalyc.org/pdf/839/83952052018.pdf

Hair, J. F., Anderson, R. E., Tatham, R.L., \& Black, W.C. (1999). Análisis multivariante. Madrid: Pearson Prentice Hall.

Heredia, Y., \& Camacho, F. (2014). Factores que afectan el desempeño académico. México: Tecnológico de Monterrey.

Huertas, A., \& Pantoja, A. (2016). Efectos de un programa educativo basado en el uso de las TIC sobre el rendimiento académico y la motivación del alumnado en la asignatura de tecnología de educación secundaria. Educación XX1, 19(2), pp. 229-250. https://doi. org/10.5944/educxx1.16464

Labrador, F., \& Villadangos, S. (2010). Menores y nuevas tecnologías: conductas indicadoras de un posible problema de adicción. Psicothema, 22(2), pp. 180-188. https://www.redalyc. org/pdf/727/72712496002.pdf

Labrador, F., Villadangos, S., Crespo, M., \& Becoña, E. (2013). Desarrollo y validación del cuestionario de uso problemático de nuevas tecnologías (UPNT). Anales de Psicología, 29(3), pp. 836-847. http://dx.doi.org/10.6018/analesps.29.3.159291

Llanga, E., Silva, M., \& Vistin, J. (2019). Motivación extrínseca e intrínseca en el estudiante, Revista Atlante: Cuadernos de Educación y Desarrollo. https://www.eumed.net/rev/ atlante/2019/09/motivacion-extrinseca-intrinseca.html

Llorca, M., Bueno, G., Viler, F., \& Diez, M. (2010). Frecuencia del uso de videojuegos y rendimiento académico. Trabajo presentado como Ponencia en el II Congreso Internacional Comunicación (Vol. 3).

Muñoz-Miralles., R., Ortega-González., R., Batala-Martínez., C., López-Morón., M., Manresa, J., \& Torán-Monserrat, P. (2013). Acceso y uso de nuevas tecnologías entre los jóvenes de educación secundaria, implicaciones en salud. Aten Primaria, 46(2), pp. 77-88. http:// dx.doi.org/10.1016/j.aprim.2013.06.001

Pauta, R. (2015). Los niños, la influencia de la televisión y el rendimiento escolar. [Tesis de Maestría no publicada]. Universidad Nacional Mayor de San Marcos. https://cybertesis. unmsm.edu.pe/handle/20.500.12672/4542

Quevedo-Blasco, R., Quevedo-Blasco, V., \& Téllez-Trani, M. (2016). Cuestionario de evaluación motivacional del proceso de aprendizaje (EMPA). European Journal of Investigation in Health, Psychology and Education, 6(2), pp. 83-105. https://doi.org/10.30552/ejihpe. 
v6i2.163

Roca, G. (2015). Las nuevas tecnologías en niños y adolescentes. Guía para educar saludablemente en una sociedad digital. Barcelona:Faros Sant Joan de Déu.

Sánchez, F. (2018). Influencia del uso del celular en el aprendizaje activo dentro del aula en estudiantes de la escuela profesional de educación primaria de la Universidad Nacional del Altiplano - Puno [Tesis de pregrado no publicada]. Universidad Nacional del Altiplano - Puno. http://repositorio.unap.edu.pe/handle/UNAP/11406

Sánchez, L., Crespo, G., Aguilar, R., Bueno, F., Benavente, R., \& Valderrama, J. (2015). Los adolescentes y las tecnologías de la información y la comunicación (TIC). Guía para padres. Valencia: Universidad de Valencia.

Sánchez-Teruel, D., \& Robles-Bello, M. (2016). Riesgos y potencialidades de la era digital para la infancia y la adolescencia. Revista Educación y Humanismo, 18(31), pp. 186-204. https://doi.org/10.17081/eduhum.18.31.1374

Solís, R., \& Valdivia, M. (2018). Uso de las TIC y su relación con la motivación para el aprendizaje en los estudiantes de la I.E. Fizcalizada Orcopampa [Tesis de maestría no publicada]. Universidad Cesar Vallejo. https://repositorio.ucv.edu.pe/handle/20.500.12692/35783

Usán, P., \& Salavera, C. (2018). Motivación escolar, inteligencia emocional y rendimiento académico en estudiantes de educación secundaria obligatoria. Actitudes en Psicología, 32(125), pp. 95-112. http://dx.doi.org/10.15517/ap.v32i125.32123

Valenzuela, J. (2007). Más allá de la tarea: Pistas para una redefinición del concepto de motivación escolar. Educaçao e Pesquisa, 33(3), pp. 409-426. https://doi.org/10.1590/ S1517-97022007000300002 